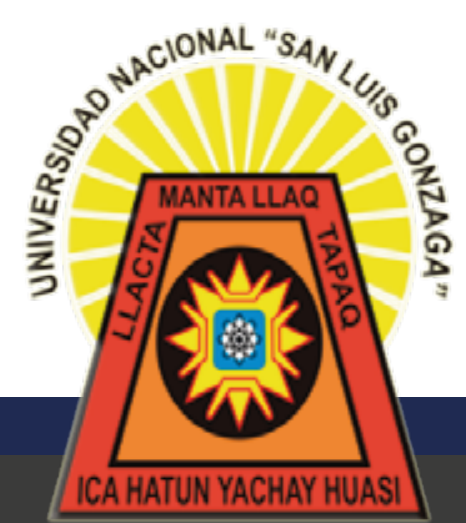

p-ISSN 2223-2893

e-ISSN 2225-6989
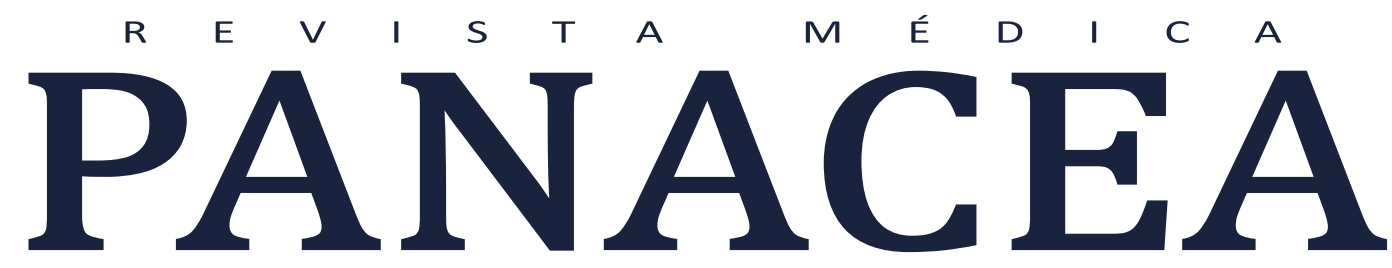

UNIVERSIDAD NACIONAL SAN LUIS GONZAGA. ICA, PERÚ

ARTÍCULO ORIGINAL:

FACULTAD DE MEDICINA HUMANA "DANIEL ALCIDES CARRIÓN"

\title{
FACTORES DE RIESGO LABORAL Y ENFERMEDADES OCUPACIONALES EN EL PROFESIONAL DE ENFERMERÍA DEL CENTRO QUIRÚRGICO HOSPITAL NACIONAL DOS DE MAYO, 2019.
}

Relationship between occupational risk factors and occupational diseases in the nursing professional who works at the surgical center of the national hospital dos de mayo, 2019.

\section{AUTOR:}

CAMACUARI CÁRDENAS FELIMAN SALOMÉ

INDEXADA EN:
$\underset{\text { catálogo }}{\operatorname{latin}}$
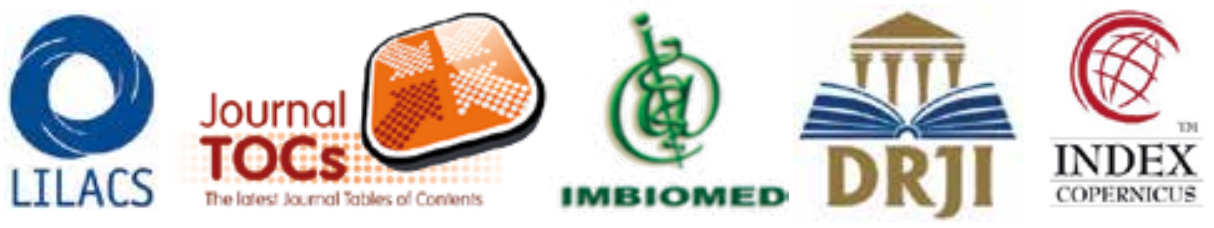
Google

\section{REVISTAS.UNICA.EDU.PE}




\section{FACTORES DE RIESGO LABORAL Y ENFERMEDADES OCUPACIONALES EN EL PROFESIONAL DE ENFERMERÍA DEL CENTRO QUIRÚRGICO HOSPITAL NACIONAL DOS DE MAYO, 2019.}

RELATIONSHIP BETWEEN OCCUPATIONAL RISK FACTORS AND OCCUPATIONAL DISEASES IN THE NURSING PROFESSIONAL WHO WORKS AT THE SURGICAL CENTER OF THE NATIONAL HOSPITAL DOS DE MAYO, 2019.

Camacuari-Cárdenas Feliman Salomé 1,a.

1. Escuela Profesional de Enfermería, Universidad Alas Peruanas. Lima, Perú.

a. Enfermera ; Licenciada en Enfermería ; Especialidad en Centro

Quirúrgico ; Maestría en Gestión en Enfermería

DOI: https://doi.org/10.35563/rmp.v10i2.431

Correspondencia:

Feliman Salomé Camacuari Cárdenas

Dirección: Cerro Bello Manzana F Lote 15, Santiago de Surco. Lima, Perú.

Correo electrónico:

salome.2111@gmail.com

Celular: 993580017

Contribuciones de autoría: FSCC: Participó en la concepción y diseño del manuscrito, recolección, análisis e interpretación de los datos, redacción y revisión crítica del contenido del manuscrito y aprobación final del artículo.

Conflicto de intereses: no existen conflictos de intereses del autor o autores de orden económico, institucional, laboral o personal.

\section{Financiamiento:}

Autofinanciado.

\section{Cómo citar:}

Camacuari - Cárdenas F. factores de riesgo laboral y e $\mathrm{n} f \mathrm{e} r \mathrm{~m}$ e $\mathrm{d}$ a d e $\mathrm{s}$ ocupacionales en el profesional de enfermería del centro quirúrgico Hospital Nacional Dos de Mayo, 2019.

Rev méd panacea 2021;10(2): 89-93. DOI: https://doi.org/10.35563 /rmp.v10i2.431

\section{RESUMEN}

Objetivo: Determinar la relación entre los factores de riesgo laboral y las enfermedades ocupacionales en el profesional de enfermería que labora en el Centro Quirúrgico del Hospital Nacional Dos de Mayo, 2019. Materiales y métodos: El método fue de tipo cuantitativo, nivel descriptivo, método de corte transversal y diseño correlacional. La población estuvo conformada por los 30 profesionales de enfermería. La técnica fue la encuesta y el instrumento un cuestionario. El instrumento utilizado fue validado por el alfa de Cronbach $(a=0,82)$. Resultados: De los 30 profesionales de enfermería, en cuanto a la primera variable acerca de los factores de riesgo laboral están presentes 66,7\% (20/30) y ausentes 33,3\% (10/30); según dimensiones biológico están presentes $66,7 \%(20 / 30)$ y ausentes 33,3\% (10/30), y ergonómico están presentes $70,0 \%$ $(21 / 30)$ y ausentes $30,0 \%(09 / 30)$; y con referencia a la segunda variable sobre las enfermedades ocupacionales presentan $43,3 \%(13 / 30)$ y no presentan $23,3 \%(07 / 30)$; según dimensiones enfermedad infecciosa no presentan $83,3 \%$ (25/30) y presentan $16,7 \%(05 / 30)$, y enfermedad músculo esquelética presentan 96,7\% (29/30) y no presentan 3,3\% (01/30); con un coeficiente de correlación Rho de Spearman de 0,738 , p-valor 0,023 , con un nivel de significancia de 0,95 y a $=0,05$. Conclusiones: Se ha demostrado que existe relación entre el factor de riesgo laboral y las enfermedades ocupacionales en los profesionales de enfermería que laboran en el Centro Quirúrgico del Hospital Nacional Dos de Mayo, 2019.

Palabras clave: Riesgo laboral, enfermedades ocupacionales, profesional de enfermería, ergonómico.

\section{ABSTRACT}

Objective: To determine the relationship between occupational risk factors and occupational diseases in the nursing professional who works at the Surgical Center of the National Hospital Dos de Mayo, 2019. Material and methods: The method was of a quantitative type, descriptive level, method cross-sectional and correlational design. The population was made up of 30 nursing professionals. The technique was the survey and the instrument a questionnaire. The instrument used was validated by Cronbach's alpha $(a=0.82)$. Results: Of the 30 nursing professionals, regarding the first variable regarding occupational risk factors, $66.7 \%(20 / 30)$ are present and 33.3\% (10/30) are absent; according to biological dimensions, $66.7 \%(20 / 30)$ are present and 33.3\% (10/30) are absent, and ergonomic, 70.0\% (21/30) and 30.0\% (09/30) are absent; and with reference to the second variable on occupational diseases they present $43,3 \%(13 / 30)$ and they do not present 23.3\% (07/30); according to infectious disease dimensions, they did not present $83.3 \%(25 / 30)$ and they presented $16.7 \%(05 / 30)$, and musculoskeletal disease presented $96.7 \%(29 / 30)$ and they did not present $3.3 \%(01 / 30)$; with a Spearman's Rho correlation coefficient of 0.738 , p-value 0.023 , with a significance level of 0.95 and $a=0.05$. Conclusions: It has been shown that there is a relationship between the occupational risk factor and occupational diseases in nursing professionals who work at the Surgical Center of the National Hospital Dos de Mayo, 2019.

Key words: Occupational risk, occupational diseases, nursing professional, ergonomic. 
Factores de riesgo laboral y enfermedades ocupacionales en el profesional de enfermería del Centro Quirúrgico Hospital Nacional Dos de Mayo, 2019.

\section{INTRODUCCIÓN}

El riesgo laboral es la posible ocurrencia de que reporte un daño o evento durante su jornada laboral; se denomina de gravedad cuando sea posible se concrete en un accidente laboral alto, teniendo como consecuencia graves daños en el aspecto físico, social o mental; por ende, las enfermeras(os) en su ambiente laboral están exponiéndose de riesgos laborales; asimismo, muchas veces pueden afrontar riesgo de contagio con pacientes durante su cuidado, y sufrir lesiones músculo esqueléticas durante su ejercicio laboral (1).

En la investigación titulada: "Factores de riesgo laboral y las enfermedades ocupacionales en el profesional de enfermería que labora en el Centro Quirúrgico del Hospital Nacional Dos de Mayo, 2019"; teniendo en consideración que el centro quirúrgico ha evolucionado ostensiblemente en todo el mundo, ocasionando una extensa serie de riesgos tanto biológicos y ergonómicos, que son generadores de enfermedades ocupacionales (2) en las enfermeras(os); partiendo de este hecho se considera desde un aspecto hipotético como riesgo laboral, al problema que se encuentra expuesto todo trabajador de salud, especialmente de enfermería, trayendo consigo a largo plazo consecuencias en el ámbito laboral, con la consecuente enfermedad ocupacional; asimismo, la identificación de los factores de riesgos laborales en enfermería permitirá la adopción de medidas correctivas para la prevención de enfermedades ocupacionales $(3,4,5)$.

El objetivo de esta investigación fue determinar la relación entre los factores de riesgo laboral y las enfermedades ocupacionales en el profesional de enfermería que labora en el Centro Quirúrgico del Hospital Nacional Dos de Mayo (HMNDM).

\section{MATERIALES Y MÉTODOS}

El estudio fue de tipo analítico, descriptivo de corte transversal $(6,7)$; asimismo, fue prospectivo, ya que se realizó en el presente, y además fue no experimental ya que no se realizó ninguna manipulación de las variables de estudio se trabajó tal y como se presentó.

El tamaño de la muestra estuvo constituido por 30 profesionales de enfermería.

La selección de la muestra fue la siguiente:

- Enfermeros(as) que laboran en el Centro Quirúrgico del HMNDM.

- Sala de operaciones del hospital.

- Que hayan trabajado un tiempo mayor a un 1 año.

- Que hayan aceptado participar voluntariamente en el estudio previo consentimiento informado.

Se utilizó en el presente estudio la encuesta y como instrumentos un cuestionario y guía de observación estructurado acerca de la relación entre los factores de riesgo laboral y las enfermedades ocupacionales en el profesional de enfermería que labora en el Centro Quirúrgico del Hospital Nacional Dos de Mayo, 2019.

Los instrumentos usados fueron revisados por profesionales de enfermería expertos, para darle las correcciones dadas por jueces y presentarlo en la tabla de Prueba Binomial $(p=0,0298)$. Posteriormente se calculó la Prueba Estadística de confiabilidad del instrumento; denominado la prueba de Alpha de Cronbach $(a=0,86)$.

Se llevó a cabo el trámite administrativo con dos oficios dirigidos al director de la institución; siendo los documentos para la autorización y aprobación para realizar el estudio, cuyo registro es $\mathrm{N}^{\circ}$ 014638, con Informe $\mathrm{N}^{\circ} 9$-OACDI-HNDM de la Oficina de Apoyo a la Capacitación, Docencia e Investigación del Hospital Nacional Dos de Mayo, y la Evaluación $\mathrm{N}^{\circ}$ 070-2019-CEIB-HNDM del Comité de Ética e Investigación Biomédica, que fueron aprobados favorablemente. Luego se coordinó con la Enfermera Jefa de Centro Quirúrgico, para realizar el levantamiento de datos.

Una vez recolectado los datos, éstos fueron llevados a cabo previa elaboración de la tabla matriz de datos generales y específicos de la variable de estudio, donde los hallazgos fueron plasmados en gráficos de barras o círculos, para analizarlos e interpretarlos en base al marco teórico o base teórica. Para la medición de la variable se aplicó el modelo de Estanones (para calcular el valor final de las variables de factores de riesgo laboral ausente y presente; y para las enfermedades ocupacionales no presenta y presenta) (Orlandoni. Escalas de medición en Estadística. Maracaibo: Universidad Privada Dr. Rafael Belloso Chacin; 2015).

Asimismo, para establecer la relación o asociación entre variables de estudio se utilizó la Prueba de Correlación Rho de Spearman, con p-valor menor que 0,05 y nivel de la confiabilidad del 0.95, para reportarlos en las tablas de contingencia o inferencial entre ambas.

Se tomó en cuenta el permiso del director del Hospital Nacional Dos de Mayo y el consentimiento informado de los profesionales de enfermería sujetos en el estudio. Asimismo, los datos fueron utilizados solo con fines de la presente investigación. (Código de Ética de la Investigación de la Universidad Nacional Mayor de San Marcos N 02996-SG-17; 2017).

\section{RESULTADOS}

Una vez concluido el procesamiento de la información, se procedió a presentar los resultados en gráficos estadísticos para su respectivo análisis e interpretación.

Con respecto a los datos generales; del 100,0\% (30/30); el 73,3\% (22/30) tienen de 25 a 39 años, 20,0\% (06/30) de 40 a 59 años, y $6,7 \%(02 / 30)$ de 50 a más años; el $73,3 \%$ (22) son mujeres, y $26,7 \%(08 / 30)$ varones; el $66,7 \%(20 / 30)$ son casados y el $33,3 \%$ $(10 / 30)$ solteros; con un tiempo de servicio en el Centro Quirúrgico de más de 10 años 53,3\% (16/30), de 2 a 5 años 30,0\% (09/30), y de 6 a 10 años 16,7\% (05/30); con un tiempo laboral en el Hospital Dos de Mayo de 43,3\% (13/30) de 6 a 10 años, 26,7\% (08/30) con más de 10 años, 20,0\% (06/30) de 2 a 5 años, y 10,0\% (03/30) con menos de 1 año en la institución.

Por lo tanto, se puede determinar que la mayoría de los profesionales de enfermería que laboran en el Hospital Nacional Dos de Mayo; tienen edades comprendidas entre 25 a 39 años, son de sexo femenino, son casados, vienen laborando en el Servicio de Centro Quirúrgico más de 10 años, y laborando en el Hospital Dos de Mayo un tiempo de 6 a 10 años.

En la tabla 1, se puede evidenciar que cuando los factores de riesgo laborales están ausentes no presentan enfermedades 
ocupacionales en un 20,0\% (06/30); y cuando los factores de riesgo laboral están presentes presentan enfermedades ocupacionales $43,3 \%(13 / 30)$.

Tabla 1. Factores de riesgo laboral y enfermedades ocupacionales en el profesional de enfermería que labora en el Centro Quirúrgico del Hospital Nacional Dos de Mayo, 2019.

\begin{tabular}{|c|c|c|c|c|c|c|}
\hline \multirow{3}{*}{ Factores de Riesgo Laboral } & \multicolumn{4}{|c|}{ Enfermedades Ocupacionales } & \multirow{2}{*}{\multicolumn{2}{|c|}{ Total }} \\
\hline & \multicolumn{2}{|c|}{ No presenta } & \multicolumn{2}{|c|}{ Presenta } & & \\
\hline & $\mathbf{n}$ & $\%$ & $\mathbf{n}$ & $\%$ & $\mathbf{n}$ & $\%$ \\
\hline Ausentes & 6 & 20.0 & 4 & 13.3 & 10 & 33.3 \\
\hline Presentes & 7 & 23.3 & 13 & 43.3 & 20 & 66.7 \\
\hline Total & 13 & 43.3 & 17 & 56.7 & 30 & 100.0 \\
\hline
\end{tabular}

Fuente: Elaboración propia utilizando el Programa SPSS 25.0

En la tabla 2, con respecto a los factores de riesgo laboral en la dimensión biológico se puede observar que están presentes en un $66,7 \%(20 / 30)$, y ausentes $33,3 \%(10 / 30)$; en cuanto a la dimensión ergonómico los factores de riesgo laboral están presentes $70,0 \%(21 / 30)$ y ausentes $30,0 \%(09 / 30)$.

Tabla 2. Factores de riesgo laboral en la dimensión biológico y ergonómico en el profesional de enfermería que labora en el Centro Quirúrgico del Hospital Nacional Dos de Mayo, 2019.

\begin{tabular}{lcccc} 
Factores de Riesgo Laboral & \multicolumn{2}{c}{ Biológico } & \multicolumn{2}{c}{ Ergonómico } \\
& $\mathbf{n}$ & $\%$ & $\mathbf{n}$ & $\%$ \\
Ausentes & 10 & 33.3 & 9 & 30.0 \\
Presentes & 20 & 66.7 & 21 & 70.0 \\
Total & 30 & 100.0 & 30 & 100.0 \\
\hline Fuente: Elaboración propia utilizando
\end{tabular}

En la tabla 3, con respecto a la dimensión enfermedad infecciosa se puede observar que no se presentan en un $83,3 \%(25 / 30)$ y presentan 16,7\% (05/30); en cuanto a la dimensión enfermedad músculo esquelética están presentes en un $96,7 \%$ (29/30), y no presentan en un 3,3\% (01/30).

Tabla 3. Enfermedades ocupacionales en la dimensión infecciosa y músculo esquelética en el profesional de enfermería que labora en el Centro Quirúrgico del Hospital Nacional Dos de Mayo, 2019.

\begin{tabular}{lcccc}
\multicolumn{1}{r}{$\begin{array}{c}\text { Enfermedades } \\
\text { Ocupacionales }\end{array}$} & \multicolumn{2}{c}{ Infecciosa } & \multicolumn{2}{c}{ Músculo esquelético } \\
No Presenta & 25 & 83.3 & 1 & 03.3 \\
Presenta & 5 & 16.7 & 29 & 96.7 \\
Total & 30 & 100.0 & 30 & 100.0 \\
\hline
\end{tabular}

Fuente: Elaboración propia utilizando el Programa SPSS 25.0

\section{DISCUSIÓN}

Los profesionales de enfermería que laboran en el Centro Quirúrgico del Hospital Nacional Dos de Mayo; con respecto a los factores de riesgo laboral en la dimensión biológico (8), se puede observar que están presentes porque manipulan objetos punzocortantes, materiales con sangre y fluidos corporales, contacto con piel y mucosas se exponen a salpicaduras por agentes activos frente al VIH y VHB $(9,10)$; y están ausentes porque se lavan las manos con clorhexidina al 4,0\% antes y después de la cirugía, hacen el uso correcto de la mascarilla, los mandilones, se colocan las botas antes del ingreso a cirugía, usan los protectores oculares, cuentan con una correcta eliminación de las agujas, eliminan el material con sangre y fluidos en bolsas rojas (tabla 1).

Se ha reportado hallazgos coincidentes donde los profesionales de enfermería que laboran en los centros quirúrgicos presentan un riesgo biológico elevado por estar en contacto directo con sangre y fluidos corporales, seguido de un menor porcentaje por no hacer uso de las barreras de protección durante la cirugía $(11,12)$.
El riesgo biológico es la infección aguda o crónica, cuya infección son causales por bacterias, virus, clamidias u hongos; es el que más presentan los profesionales de enfermería; en contacto con la sangre o fluidos corporales, pinchazos por objetos punzocortantes, contacto con piel y mucosas; asimismo, estar expuestos de microorganismos, mayor susceptibilidad de generar infecciones en las(os) enfermeras(os), otra de las fuentes son los humificadores; todos los sistemas de canalización del agua, y los equipos de refrigeración, conllevando a propagarse cantidad de microorganismos que estos producen riesgos tóxicos o irritantes; con ello traen consecuencia ocasionados por los riesgos biológicos como principales agentes biológicos con mayor riesgo son las infecciones de la hepatitis $B$, hepatitis $C$, y VIH/SIDA, entre otros (13).

Los profesionales de enfermería que laboran en el Centro Quirúrgico del Hospital Nacional Dos de Mayo; en cuanto a la dimensión ergonómico (14) los factores de riesgo laboral están presentes porque suelen levantar objetos que pesan más de 12 Kilos, levantan objetos por encima de 1,8 metros de altura, gira el tronco al elevar la carga o transportarla (torsión), manipula cargas muy cerca del suelo (agachado), el entorno donde se levantan las cargas es inadecuado, mantiene posturas estáticas durante la cirugía, tronco flexionado y girado, mantienen las rodillas flexionadas con el peso del cuerpo, paran de pie tiempos prolongados y mantienen el cuello flexionado el 50,0\% de duración de tarea; y están ausentes porque evitan trabajar uno o ambos brazos por encima de los hombros, movimientos de brazos, manos y muñecas no repetidos y nada de esfuerzo intenso (tabla 2 ).

En el estudio se reportaron hallazgos coincidentes donde el riesgo ergonómico en enfermeras influye en la salud ocupacional de enfermería, reportó mayormente una sobrecarga física, esfuerzo físico y postural poco más de la mitad; poco más de la mitad requerimientos excesivos de fuerza promedio, de movimiento promedio y condición inadecuada de los puestos de trabajo (15).

Los riesgos ergonómicos, por su tarea en ambientes y mobiliarios inadecuados, exposición frecuente de carga física, malas posturas, causan lesiones músculo esqueléticas, patologías que se dan con frecuencia; por ende, la causalidad de la presencia de una lesión, por unas posturas inadecuadas y forzadas, movimientos repetitivos, manipulación de cargas o pacientes, por ello, las negativas ergonomía; como una lesión de los músculos, tendones, caderas, piernas; produciendo dolor lumbar en las(os) enfermeras(os) en la institución de salud, siendo las causales de ausentarse al trabajo; aparecen por la manipulación de cargas, movimientos forzados, movimientos imprevistos, uso de pantalla de visualización de datos y movimiento de enfermos (16).

Los profesionales de enfermería que laboran en el Centro Quirúrgico del Hospital Nacional Dos de Mayo; con respecto a la dimensión enfermedad infecciosa (17), se puede observar que no se presentan porque no tienen infecciones por contagio de Virus de Hepatitis B, infección por contagio de Virus de Hepatitis $C$, e infección por contagio de virus de la inmunodeficiencia humana; y presentan enfermedades ocupacionales por infección por contagio de TB (tabla 2).

En el estudio se reportaron hallazgos coincidentes donde los profesionales de enfermería que laboran en los centros 

de Mayo, 2019.

quirúrgicos están expuestos a enfermedades infecciosas como pinchazos, y a enfermedades músculo esquelética contagios de enfermedades, luxaciones de columna, infecciones cruzadas, nosocomiales, y alergias. En otro estudio se reportó hallazgos coincidentes ya que los enfermeros encontraron agentes infecciosos y causantes de enfermedades infecciosas como $\mathrm{VIH}$, la Hepatitis B, Hepatitis $C$, un porcentaje muy elevado utiliza medidas de protección como los guantes y la mascarilla, siendo las gafas las que se utilizan con menor frecuencia (18).

El profesional de enfermería está expuesto a todo tipo de agentes biológicos y ergonómicos en los centros quirúrgicos, pudiendo contraer las enfermedades infecciosas que se ven expuestos con mayor frecuencia en su práctica diaria son las de etiología vírica, resaltando entre ellas los virus de la Hepatitis $B$ y $C$ y el VIH/SIDA; es decir, señaló que las enfermedades infecciosas son causadas por microorganismos patógenos como las bacterias, virus, y parásitos; estas enfermedades se pueden trasmitir directa o indirectamente de una persona a otra, cada enfermedad infecciosa tiene sus signos y síntomas específicos, son frecuentes en muchas enfermedades infecciosas se incluyen: fiebre, diarrea, fatiga, dolores musculares, tos, entre otros; por ende, el personal de enfermería puede estar expuesto a todo tipo de agentes biológicos $(12,19)$.

Los profesionales de enfermería que laboran en el Centro Quirúrgico del Hospital Nacional Dos de Mayo; con respecto a la dimensión enfermedad músculo esquelética están presentes porque tienen síndrome de contusión del hombro, luxación de hombro, subluxación anterior del hombro, epicondilitis humeral lateral, epicondilitis medial o síndrome del flexor pronador, tendinitis, tenosinovitis, síndrome del túnel del Carpio, bursitis, lesiones de los ligamentos de la rodilla, esguince de tobillo, cuello y espalda, cervicalgia, dorsalgia y lumbalgia; y no presentan porque no tienen síndrome del túnel radial y no sufren de bursitis prepatelar o infrapatelar las enfermeras(os) (tabla 3).

Asimismo, en el estudio se reportaron hallazgos coincidentes donde los profesionales de enfermería que laboran en los centros quirúrgicos están expuestos a enfermedades músculo esqueléticas aproximadamente la mitad de enfermeras presentó molestias físicas en la espalda en el último año, existe asociación significativa entre el dolor que presentan en espalda y mano-muñeca derecha con el riesgo de carga física sometidos $(15,20)$.

La enfermedad músculo esquelética son alteraciones que sufren estructuras corporales como los músculos, articulaciones, tendones, ligamentos, nervios, huesos y el sistema circulatorio, causadas o agravadas fundamentalmente por el trabajo y los efectos del entorno, son trastornos acumulativos resultantes por exposición repetida, durante un período de tiempo prolongado, a factores de riesgo biomecánico y organizacionales, tales trastornos afectan principalmente la espalda, cuello, hombros y extremidades superiores, también pueden afectar a las inferiores; es decir, los diagnósticos más frecuentes son las tendinitis, síndrome del túnel carpiano, lumbalgias, etc.; asimismo, normalmente afectan a la espalda, cuello, hombros y extremidades superiores, aunque también pueden afectar a las extremidades inferiores, comprenden cualquier daño o trastorno de las articulaciones y otros tejidos $(6,21)$. 


\section{REFERENCIAS BIBLIOGRÁFICAS}

1. Blanco L. Actitud en bioseguridad y exposición a riesgo laboral en enfermeras(os) en el Hospital de Apoyo Chepén. Tesis de Grado. Chepén: Universidad Nacional de Trujillo; 2019. (Citado el 10 de junio del 2019) Disponible en: http://dspace.unitru.edu.pe/handle/UNITRU/11618

2. Balseca R, Villamar S. Riesgos ocupacionales en los/las profesionales de enfermería que laboran en el hospital Dr. Abel Gilbert Pontón. Guayaquil: Universidad de Guayaquil. 2017. 1(2):01-83. (Citado el 10 de junio del 2019) Disponible en: http://repositorio.ug.edu.ec/handle/redug/3551

3. Consejo Internacional de Enfermería. Prevención de los riesgos laborales en el quirófano. Ginebra: CIE; 2018; (Citado el 10 de junio del 2019) Disponible en: https://www.icn.ch/es

4. Díaz R, Tapia S. Riesgos laborales a los que está expuesto el personal de enfermería que labora en el área quirúrgica del Hospital General Dr. Raymundo Abarca Alarcón. Chilpancingo: Instituto de Ciencias y Estudios Superiores de Tamaulipas; 2017. (Citado el 10 de junio del 2019) Disponible en:

(https://es.slideshare.net/franki23/riesgos-laborales-en-el-p ersonal-de-enfermera-en-el-rea-quirrgica-del-hospital-gen eral-dr-raymundo-abarca-alarcn

5. Espín E, Sarabia K. Riesgos laborales que desencadenan las enfermedades profesionales en el personal de enfermería, del Hospital General Docente Riobamba. Riobanba: Universidad Nacional de Chimborazo de Ecuador; 2018. (Citado el 10 de junio del 2019) Disponible en: http://dspace.unach.edu.ec/handle/51000/5429

6. Avendaño C, Grau P. Riesgos para la salud de las enfermeras del sector público. Santiago de Chile: Universidad Nacional de Chile. 2016; 10(1):1-83. (Citado el 10 de junio del 2019) Disponible en:

http://www.psykhe.cl/index.php/psykhe/article/download/1 20

7. Fang M. Percepción del personal de enfermería sobre los riesgos biológicos. Revista CONAMED. 2016; 20(01):12-16. (Citado el 10 de junio del 2019) Disponible en: https://www.medigraphic.com/cgi-bin/new/resumen.cgi?ID ARTICULO $=57382$

8. Harrison. Principios de medicina interna. Consideraciones básicas en las enfermedades infecciosas. 19 ediciónMcGraw-Hill; Interamericana de España; 2018.

9. Ministerio de Salud del Perú. Exposición ocupacional en los centros hospitalarios. Lima: MINSA; 2018; (Citado el 10 de junio del 2019) Disponible en:https://www.gob.pe/minsa/

10. Organización Mundial de la Salud. Estrategia mundial sobre salud ocupacional: camino a la salud en el trabajo. Suiza, Ginebra: OMS; 2018. (Citado el 10 de junio del 2019) Disponible en: https://www.who.int/es

11. Borda A. Accidentes de trabajo: prevalencia y factores de riesgo en enfermeras que laboran en hospitales nacionales de ESSALUD. Revista Peruana de Obstetricia y Enfermería. 2018; 8(5):1-11. (Citado el 10 de junio del 2019) Disponible en:

https://www.aulavirtualusmp.pe/ojs/index.php/rpoe/article/ view/677/530

12. Espinoza C. Personal de enfermería: Sala de Operaciones, Emergencia, Unidad de Cuidados Intensivos del Hospital Félix Torrealva. Ica: U. N. San Luis Gonzaga de Ica; 2016; 1(2):01-66. Disponible en: (Citado el 10 de junio del 2019) Disponible en:

https://www.unica.edu.pe/enfermeria/archivos/revista-enfer meria-alavanguardia-volumen2.pdf
13. Junco R. Riesgo ocupacional por exposición a objetos corto punzantes en trabajadores de la salud. Rev Cubana; 2017; 4(1):1-10. (Citado el 10 de junio del 2019) Disponible en: http://scielo.sld.cu/scielo.php?script=sci_arttext\&pid=S1561 $-30032003000100005$

14. Montalvo A, Cortés Y, Rojas M. Riesgo ergonómico asociado a sintomatología músculo esquelética en personal de enfermería. Cartagena: Universidad de Cartagena; 2017; 20(2):132-146. (Citado el 10 de junio del 2019) Disponible en: http://www.scielo.org.co/pdf/hpsal/v20n2/v20n2a10.pdf

15. Tipán D. Riesgos ergonómicos en el personal de enfermería. Valladolid: Universidad de Valladolid de España; 2018; (Citado el 10 de junio del 2019) Disponible en: http://uvadoc.uva.es/bitstream/handle/10324/32044/TFG-L 2141. pdf;jsessionid =79C295F4BB9A85AA97605DC526E2F10 8 ? sequence $=1$

16. Leyva M, Reyes I, Rosas J. Evaluación de riesgos del personal de enfermería en área quirúrgica como una necesidad permanente. Chiapas: Universidad Autónoma de Chiapas; 2018; 2(1):1-22. (Citado el 10 de junio del 2019) Disponible en:

https://www.revista-portalesmedicos.com/revista-medica/ri esgos-personal-de-enfermeria/

17. Organización Internacional del Trabajo y la Organización Mundial de la Salud. El número de accidentes y enfermedades relacionados con el trabajo sigue aumentando OIT y OMS recomiendan aplicar estrategias de prevención. Ginebra: OIT/OMS; 2018; (Citado el 10 de junio del 2019) Disponible en: https://www.ilo.org/global/lang--es/index.htm

18. Maylle. Factores de riesgo y los accidentes laborales en enfermería de un Hospital Público del Cercado de Lima, en una muestra de 40 profesionales de enfermería. Tesis de Grado. Lima: Universidad César Vallejo; 2018; (Citado el 10 de junio del 2019) Disponible en: http://repositorio.ucv.edu.pe/handle/UCV/31985?show=full

19. Jurado K. Exposición a riesgos laborales del personal de enfermería en Sala de Operaciones del Hospital III Emergencias Grau. Tesis de Grado. Lima: Universidad Nacional Mayor de San Marcos; 2017. (Citado el 10 de junio del 2019) Disponible en:http://cybertesis.unmsm.edu.pe/handle/cybertesis/7344

20. Instituto Nacional de Prevención de la Salud y de Seguridad Laboral. Enfermedades ocupacionales frente a los riesgos laborales en el ámbito hospitalario. Washington: INPSASEL; 2018. (Citado el 10 de junio del 2019) Disponible en: https://web.ins.gob.pe/es/acerca-del-ins/seguridad-y-salud -ocupacional

21. Morán C. Riesgo laboral del profesional en los quirófanos, Hospital Sergio Enrique Bernales de Collique. Lima: Universidad de San Martín de Porres; 2016. (Citado el 10 de junio del 2019) Disponible en: http://www.aulavirtualusmp.pe/ojs/index.php/rpoe/article/v iew/638

PANACEA 